\title{
The role of stimulus complexity, spectral overlap, and pitch for gap-detection thresholds in young and old listeners
}

Link to publication record in Manchester Research Explorer

Citation for published version (APA):

Heinrich, A., de la Rosa, S., \& Schneider, B. (2014). The role of stimulus complexity, spectral overlap, and pitch for gap-detection thresholds in young and old listeners. The Journal of the Acoustical Society of America, 136(4), 1797-1807.

\section{Published in:}

The Journal of the Acoustical Society of America

\section{Citing this paper}

Please note that where the full-text provided on Manchester Research Explorer is the Author Accepted Manuscript or Proof version this may differ from the final Published version. If citing, it is advised that you check and use the publisher's definitive version.

\section{General rights}

Copyright and moral rights for the publications made accessible in the Research Explorer are retained by the authors and/or other copyright owners and it is a condition of accessing publications that users recognise and abide by the legal requirements associated with these rights.

\section{Takedown policy}

If you believe that this document breaches copyright please refer to the University of Manchester's Takedown Procedures [http://man.ac.uk/04Y6Bo] or contact uml.scholarlycommunications@manchester.ac.uk providing relevant details, so we can investigate your claim.

\section{OPEN ACCESS}




\section{The role of stimulus complexity, spectral overlap, and pitch for gap-detection thresholds in young and old listeners}

A. Heinrich, S. de la Rosa, and B. A. Schneider

Citation: The Journal of the Acoustical Society of America 136, 1797 (2014); doi: 10.1121/1.4894788

View online: https://doi.org/10.1121/1.4894788

View Table of Contents: http://asa.scitation.org/toc/jas/136/4

Published by the Acoustical Society of America

\section{Articles you may be interested in}

Reference hearing thresholds in an extended frequency range as a function of age

The Journal of the Acoustical Society of America 136, 1821 (2014); 10.1121/1.4894719

Detection thresholds for gaps, overlaps, and no-gap-no-overlaps

The Journal of the Acoustical Society of America 130, 508 (2011); 10.1121/1.3598457

Development of a quick speech-in-noise test for measuring signal-to-noise ratio loss in normal-hearing and hearing-impaired listeners

The Journal of the Acoustical Society of America 116, 2395 (2004); 10.1121/1.1784440

Effects of age and frequency disparity on gap discrimination

The Journal of the Acoustical Society of America 111, 2793 (2002); 10.1121/1.1476685

Detection of temporal gaps in sinusoids by elderly subjects with and without hearing loss

The Journal of the Acoustical Society of America 92, 1923 (1992); 10.1121/1.405240 


\title{
The role of stimulus complexity, spectral overlap, and pitch for gap-detection thresholds in young and old listeners
}

\author{
A. Heinrich, ${ }^{\text {a) }}$ S. de la Rosa, ${ }^{\text {b) }}$ and B. A. Schneider \\ Human Communication Laboratory, Department of Psychology, University of Toronto Mississauga, \\ 3359 Mississauga Road North, Mississauga, Ontario L5L 1C6, Canada
}

(Received 16 December 2013; revised 8 August 2014; accepted 15 August 2014)

\begin{abstract}
Thresholds for detecting a gap between two complex tones were determined for young listeners with normal hearing and old listeners with mild age-related hearing loss. The leading tonal marker was always a $20-\mathrm{ms}, 250-\mathrm{Hz}$ complex tone with energy at $250,500,750$, and $1000 \mathrm{~Hz}$. The lagging marker, also tonal, could differ from the leading marker with respect to fundamental frequency (f0), the presence versus absence of energy at f0, and the degree to which it overlapped spectrally with the leading marker. All stimuli were presented with steeper $(1 \mathrm{~ms})$ and less steep $(4 \mathrm{~ms})$ envelope rise and fall times. F0 differences, decreases in the degree of spectral overlap between the markers, and shallower envelope shape all contributed to increases in gap-detection thresholds. Age differences for gap detection of complex sounds were generally small and constant when gapdetection thresholds were measured on a log scale. When comparing the results for complex sounds to thresholds obtained for pure-tones in a previous study by Heinrich and Schneider [(2006). J. Acoust. Soc. Am. 119, 2316-2326], thresholds increased in an orderly fashion from markers with identical (within-channel) pure tones to different (between-channel) pure tones to complex sounds. This pattern of results was true for listeners of both ages although younger listeners had smaller thresholds overall. @ 2014 Acoustical Society of America. [http://dx.doi.org/10.1121/1.4894788]
\end{abstract}

PACS number(s): 43.66.Mk, 43.66.Sr, 43.66.Hg [EB]

Pages: $1797-1807$

\section{INTRODUCTION}

Maintaining good speech intelligibility in noisy backgrounds is one of the great difficulties and barriers to social participation as people age (Committee on Hearing Bioacoustics and Biomechanics, 1988; Frisina and Frisina, 1997; Cacciatore et al., 1999). Although the nature of the age-related changes responsible for these difficulties are not fully understood (Schneider et al., 2010), temporal processing has been repeatedly linked to speech intelligibility in complex environments (Strouse et al., 1998; Schneider and Pichora-Fuller, 2001). One of the most frequently used measures of temporal processing is the ability to detect a brief silent period (gap) in an otherwise continuous sound. In this paper, we examined how age, f0 change, and spectral overlap between periodic markers affect gap detection.

In a gap-detection task, a listener is asked to discriminate the presence versus absence of a temporal gap between two sounds. The two sounds surrounding the gap are often referred to as the leading and lagging markers for the gap. A number of studies have shown that gap-detection thresholds increase when spectral differences between markers increase. Thresholds can be as small as $1 \mathrm{~ms}$ when the leading and lagging markers consist of pure tones of the same frequency (e.g., Schneider et al., 1994; Heinrich and Schneider, 2006). In these instances, gap detection is thought

\footnotetext{
a) Author to whom correspondence should be addressed. Current address: MRC Institute of Hearing Research, University Park, Nottingham, NG7 2RD, UK. Electronic mail: antje.heinrich@ihr.mrc.ac.uk

${ }^{b}$ Current address: Max Planck Institute for Biological Cybernetics, Spemannstrasse 38, 72076 Tübingen, Germany.
}

to depend on within-channel processing, with the decision as to whether or not a gap is present based on changes in the output of the auditory filter centered on the frequency of the pure tone. In within-channel tasks, older listeners typically show a small but consistent elevation in threshold levels compared to younger listeners with the size of the age effect decreasing as marker durations increase (Schneider and Hamstra, 1999).

In contrast, when leading and lagging markers differ in spectral content, between-channel comparisons are required to detect the gap between them, leading to substantially higher thresholds (e.g., Divenyi and Danner, 1977; Formby and Forrest, 1991; Phillips et al., 1997; Grose et al., 2001). In addition, Lister and colleagues (Lister et al., 2000; Lister et al., 2002; Lister and Tarver, 2004) showed that age differences in gap detection thresholds increased as the frequency difference between leading and lagging markers increased, indicating that frequency disparity modulated the size of the age difference in gap detection thresholds. Note that in these studies both markers typically share the same type of periodicity - either both are pure tones or both are noises.

Changing periodicity between markers (from aperiodic to periodic) also affects gap-detection thresholds. PichoraFuller et al. (2006) determined gap detection thresholds in two conditions: (1) When both leading and lagging markers were periodic (either both markers were identical pure tones or identical vowels) and (2) when the leading marker was aperiodic and the lagging marker was periodic (either noisetone or fricative-vowel as leading and lagging markers, respectively). Stimuli that changed periodicity (noise to tone or fricative to vowel) led to much higher overall thresholds than those that kept periodicity constant across markers (two 
pure tones or two vowels). Moreover, the age effect was much larger when marker periodicity changed from aperiodic to periodic than when both markers were periodic. Note that in the Pichora-Fuller et al. (2006) study, when both markers were periodic they were also identical, i.e., the detection task was within-channel. However, in their between-channel conditions, in addition to a change in spectral overlap between the first and second markers, there was also a change in periodicity with the first marker being aperiodic and the second marker periodic. Hence it is not clear whether the increased thresholds in their between-channel conditions were due to differences in spectral overlap, periodicity or a combination of both. In the current study, we investigated in younger and older adults how changes in spectral content (f0 and spectral overlap) between markers affected gap detection thresholds when periodicity was held constant because both markers were periodic. With regard to f0 and spectral overlap, Oxenham (2000) found for unresolved frequencies that changing the spectral region between complex-tone markers of identical fundamental frequency (f0) was more disruptive to gap detection than changing the f0 between markers in the same spectral region. In resolved complexes with tuned and mistuned harmonics, Leung et al. (2011) showed that mistuning any harmonic in the complex led to impaired gap detection and suggested that mistuning impaired the grouping of the harmonics into a unified object, which then impaired gap detection. There are indications that various aspects of pitch processing decline as people age (Grube et al., 2003). Older listeners are also less sensitive than younger listeners to a frequency shift in one of the harmonics in a complex tone (Alain et al., 2001). Similarly, sensorineural hearing impairment has been associated with reduced harmonic resolvability, reduced pitch salience of complex tones with low harmonics, less accurate frequency selectivity, and broadened auditory filters (Tyler et al., 1983; Moore et al., 1999; Moore and Moore, 2003; Bernstein and Oxenham, 2006). In the current study, we investigated how changes in f0 between the first and second marker, the presence versus absence of energy at $\mathrm{f} 0$ in the second marker, and the presence or absence of spectral overlap between two complex-tonal markers affected gap detection in young and older listeners.

We also examined the degree to which younger and older adults might be differentially sensitive to the amplitude envelopes used to gate the markers on and off. For identical pure tones, Schneider, Speranza, and Pichora-Fuller (1998) found that while gap detection thresholds increased as the onsets and offsets of the markers lengthened, there was no evidence that the extent of the age difference varied with the abruptness of marker onset and offset. Here we compared two different degrees of onset and offset ramping for nonidentical complex tones to determine whether age differences in sensitivity to changes in ramp duration might begin to emerge in a between-channel gap detection task in which the two markers were complex tones differing in either $\mathrm{f0}$ and/or their degree of overlap.

Finally, to evaluate the degree to which the effects of age on gap detection might be modulated by task complexity, we evaluated the effects of age in three gap detection situations: (1) Pure-tone, within-channel gap detection in which both of the markers were identical pure tones, (2) pure-tone, between-channel gap detection in which the markers were two pure tones separated by more than one critical band, and (3) the complex-tone, between-channel gap detection situation described in the preceding text.

\section{METHODS}

\section{A. Listeners}

Twenty young listeners (mean age: $21.0 \mathrm{yr}$; SD: 1.6; 7 males) and 20 older listeners (mean age: 71.3; SD: $=5.2 ; 10$ males) participated in the study. Young listeners were university students at the University of Toronto Mississauga. Older participants were residents of the local community. The study was approved by the ethics board of the University of Toronto. All young listeners and 18 older listeners had hearing thresholds of $30 \mathrm{~dB}$ hearing level (HL) or less from $0.25 \mathrm{kHz}$ to $3 \mathrm{kHz}$ in the test ear. The remaining two older listeners had a threshold of $35 \mathrm{~dB} \mathrm{HL}$ at one test frequency, and additionally, one of the older listener had a threshold of $40 \mathrm{~dB} \mathrm{HL}$ at $3 \mathrm{kHz}$ in the test ear. Pure-tone airconduction thresholds for all tested frequencies in the test ear (left) are displayed in Table I. Thresholds were significantly higher at and above $1 \mathrm{kHz}$ for older than for younger adults. The older adults can be characterized as being in the early stages of presbyacusis (Clark, 1981).

\section{B. Stimuli}

All stimuli were digitally constructed with a sampling rate of $20 \mathrm{kHz}$ and converted to analog form using a 16-bit Tucker-Davis Technologies (TDT) System II digital-to-analog converter. The amplitude envelopes of the markers were constructed by summing a series of Gaussian functions, which either had a standard deviation of $1 \mathrm{~ms}$ (SD1) and were spaced $1 \mathrm{~ms}$ apart, or had a standard deviation of $4 \mathrm{~ms}$ (SD4) and were spaced $4 \mathrm{~ms}$ apart. In all cases, the sum of the Gaussians formed a flat top with ogival rise and decay

TABLE I. Mean and standard deviation (SD) of audiometric thresholds in $\mathrm{dB}$ HL for all nine tested pure-tone frequencies in the left test ear for younger and older listeners. Stars denote significant differences in hearing level between the age groups.

\begin{tabular}{|c|c|c|c|c|}
\hline \multirow[b]{2}{*}{ Frequency in $\mathrm{kHz}$} & \multicolumn{2}{|c|}{ Young } & \multicolumn{2}{|c|}{ Old } \\
\hline & Mean & $\mathrm{SD}$ & Mean & $\mathrm{SD}$ \\
\hline 0.25 & 9.75 & 5.95 & 9.00 & 7.54 \\
\hline 0.5 & 5.75 & 5.91 & 7.75 & 6.97 \\
\hline 1 & 1.00 & 4.76 & $9.25^{* *}$ & 8.32 \\
\hline 1.5 & 3.25 & 4.94 & $13.00^{*}$ & 8.34 \\
\hline 2 & 1.25 & 5.59 & $16.00^{* *}$ & 9.68 \\
\hline 3 & -0.50 & 6.67 & $19.75^{* *}$ & 11.29 \\
\hline 4 & 3.75 & 5.10 & $28.75^{* *}$ & 10.75 \\
\hline 6 & 6.25 & 8.56 & $34.00^{* *}$ & 17.81 \\
\hline 8 & 3.75 & 8.25 & $37.50^{* *}$ & 21.67 \\
\hline
\end{tabular}


times. The envelopes of the SD1 and SD4 stimuli differed in the duration of the on and off ramps [Figs. 1(A) and 1(C)].

Shorter ramps increase the risk of spectral splatter as an additional cue to gap detection. However, using this technique of envelope construction, Heinrich and Schneider (2006) showed mathematically and graphically that the spectral energy of two 20-ms markers of 1 and $2 \mathrm{kHz}$ generated by the summing of Gaussians and separated or not, by a $13 \mathrm{~ms}$ gap, were essentially equal. Moreover, an application of their Eq. (A5) (which specifies the energy spectra for gap and no-gap stimuli) also shows that energy spectra of gap and no-gap stimuli are quite similar when identical in-phase pure tones are used as markers (see also Schneider et al., 1994; Schneider and Hamstra, 1999 for a similar argument). Using this technique of stimulus generation makes it unlikely that spectral splatter contributes to the discriminability between gap and no-gap stimuli, regardless of the spectral content of the two markers.

In all conditions, the duration of the leading and lagging markers bracketing the gap was $20 \mathrm{~ms}$, measured from the first to the last peak of the Gaussians of the marker envelope. The particular marker duration was chosen to facilitate comparison with the results of a previous study, which measured pure-tone between-channel gap thresholds for stimuli generated using the same technique (Heinrich and Schneider, 2006). We are aware that $20-\mathrm{ms}$ marker duration is at the lower end of tonality perception, but judging by Mohlin (2011) the duration should be long enough to perceive the pitch experienced when the complex tone is presented for a longer duration. Gaps were created by changing the envelope structure of the sound in such a way that the first Gaussian of the lagging marker was delayed by an integer number of SD relative to the last Gaussian of the leading marker. This was equivalent to omitting an integer number of Gaussians between the two markers. The smallest possible gap size constructed in this manner between the peak of last Gaussian of the leading marker and the peak of the first Gaussian of the lagging marker was $2 \mathrm{~ms}$ for the SD1 stimuli, and $8 \mathrm{~ms}$ for the SD4 stimuli. The no-gap stimuli were created by filling in the gap between markers with an even number of Gaussians. The durations of the leading and lagging markers were adjusted to match the overall duration of the gap stimulus as closely as possible, subject to the constraint that the leading and lagging markers forming the no-gap stimulus were of equal duration. Specifically, when the leading and lagging markers were separated by an even number of missing Gaussians, the duration of the no-gap stimulus precisely matched that of the gap stimulus, while when the leading and lagging markers were separated by an odd number of missing Gaussians, the duration of the no-gap stimulus exceeded that of the corresponding gap stimulus by one standard deviation [compare Figs. 1(A) and 1(B) with 1(C) and 1(D)]. As shown previously, these differences in overall duration between gap and no-gap stimuli are too small to be used by either age group as extraneous cues to gap detection (Heinrich and Schneider, 2006).

We used this technique despite the slight difference in overall stimulus duration for half of the stimuli because it preserved the phase of the sinusoidal stimuli across the gap.
Preserving the stimulus phase is essential to synchronize the stimulus phase with the ringing in the auditory filter and not cause a perturbation in the output of the filter, which in turn would create a cue that can interact with gap detection and lead to a serious underestimation of a listener's gapdetection threshold (Shailer and Moore, 1987). Last, the marker envelopes were multiplied by tone complexes representing the stimulus conditions to form the stimuli used in the experiments.

Heinrich and Schneider (2006) showed that the minimum gap of $2 \mathrm{~ms}$ was often perceivable by young listeners in a within-channel task. Because gap size could not be further reduced by omitting Gaussians, they created smaller gaps by filling the space with a reduced-amplitude Gaussian. ${ }^{1}$ Using this technique introduced a shallow dip in the stimulus envelope [compare Figs. 1(C) and 1(E)]. However, this technique also changed the overall shape of the stimulus envelope and made the half-power points of the leading and lagging marker unusable as a measure of gap duration. To use this stimulus generation technique but avoid the measurement problem, we used the area difference between gap and no-gap amplitude envelopes [Figs. 1(E) and $1(\mathrm{D})$ ] to measure gap duration and conducted all analyses on this area difference. However, in describing and plotting the data, we converted the area measures back into equivalent gap durations. ${ }^{1}$

All stimulus conditions are displayed in Fig. 2(A). All markers except those in the BASE conditions were harmonic complexes with four harmonics. The fundamental frequency (f0) of the leading marker was $250 \mathrm{~Hz}$ with energy present at the fundamental frequency, giving rise to a clear pitch of $250 \mathrm{~Hz}$. The lowest frequency in the lagging markers was $1 \mathrm{kHz}$. The lagging marker in condition SFSF had an f0 of $250 \mathrm{~Hz}$ (with no energy at f0) and a common shared frequency of $1 \mathrm{kHz}$ (same f0 shared frequency, SFSF). The lagging marker in condition SFNF also had an f0 of 250 (no energy at 250) but did not share a frequency with the leading marker (same f0 no shared frequency, SFNF). The lagging marker in condition DFSF had an $\mathrm{f0}$ of $1 \mathrm{kHz}$ (energy at f0) and hence also shared a frequency with the leading marker (different f0 shared frequency, DFSF). The lagging marker in condition DFNF had an f0 of $1 \mathrm{kHz}$ (but no energy at $1 \mathrm{kHz}$ ) and therefore no shared frequency (different f0 no shared frequency, DFNF). Lagging markers in all four conditions were perceived as having a clear pitch, which was either the same (SFSF, SFNF) as or different (DFSF, DFNF) from the leading marker. Finally, the lagging marker in condition UFNF (unclear f0 no shared frequency) with energy at $1300,1900,2100$, and $4100 \mathrm{~Hz}$ had an $\mathrm{f} 0$ of $100 \mathrm{~Hz}$ but no energy at $\mathrm{f} 0$, did not share a frequency with the leading marker and did not produce a clear pitch (Plomp, 1967; Terhardt, 1974). All stimuli were presented with envelopes that had more abrupt (1-ms SD Gaussians, SD1) or more gradual (4-ms SD Gaussians, SD4) envelope onsets and offsets. We also measured gap-detection thresholds for a condition in which both markers were $1-\mathrm{kHz}$ sinusoids [BASE (SD1) and BASE (SD4)]. The sound pressure level (SPL) of the complex tones before multiplication with the marker envelopes was $70 \mathrm{~dB}$ SPL. After multiplication, the stimuli 


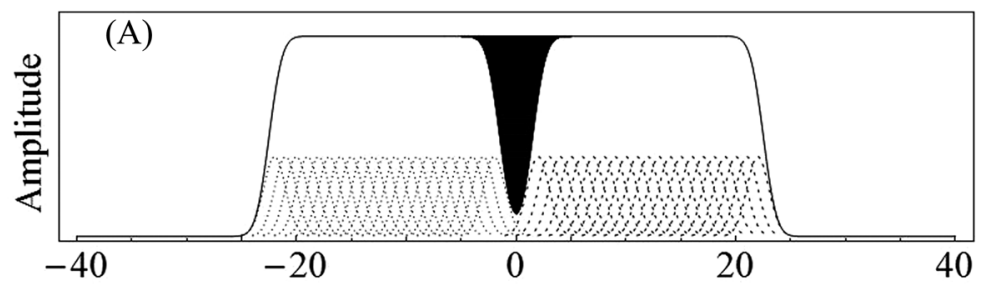

Time from stimulus center in $\mathrm{ms}$

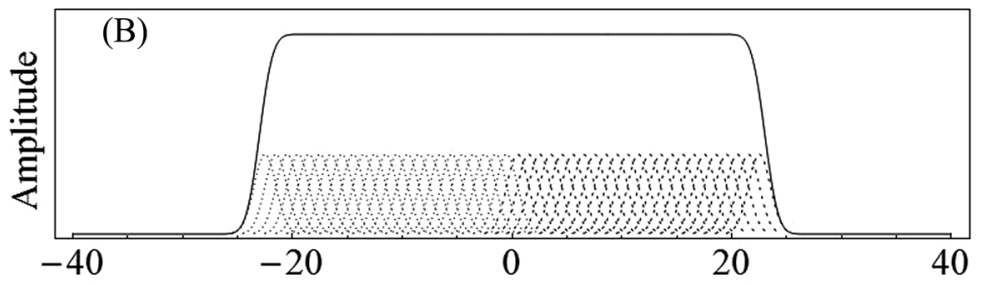

Time from stimulus center in $\mathrm{ms}$

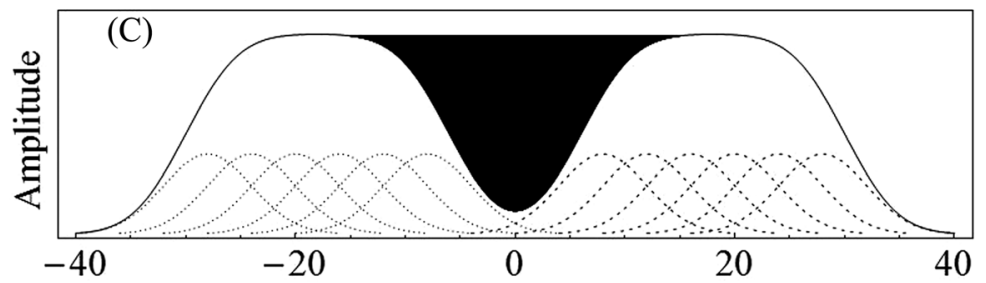

Time from stimulus center in $\mathrm{ms}$

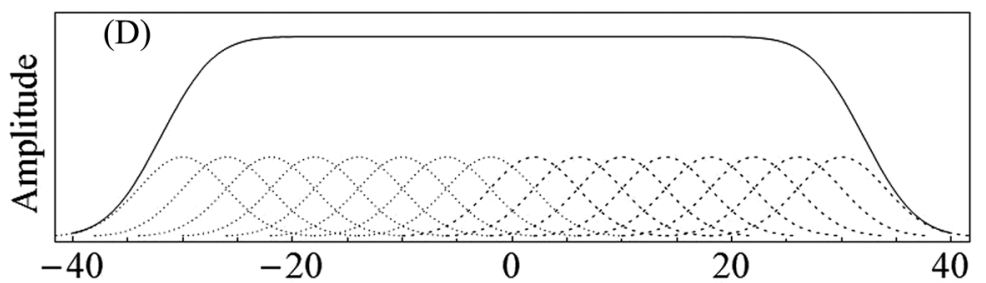

Time from stimulus center in $\mathrm{ms}$

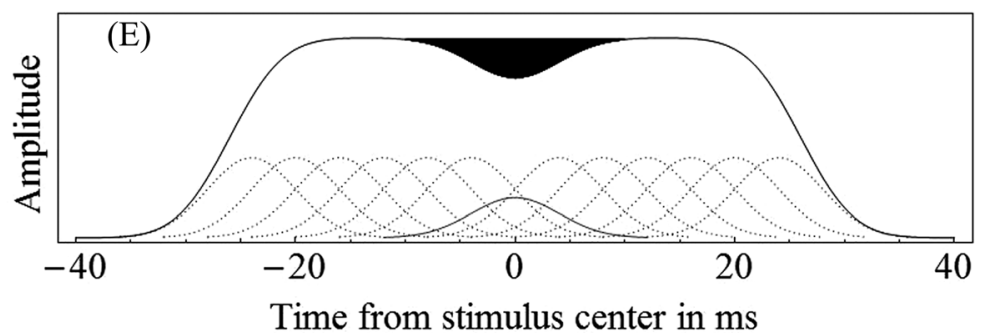

FIG. 1. (A) A total of 21 Gaussians with 1-ms standard-deviation (SD) envelopes are used to create each of two leading and lagging 20-ms marker envelopes. The markers are separated by a 4-ms gap, which corresponds to the removal of three 1-ms Gaussians. The Gaussians are spaced 1 ms apart. The Gaussians defining the first marker are indicated by the fine dotted lines. The Gaussians defining the second marker are indicated by the dashed lines. The blackened portion indicates the difference in area between the central dip in the envelope and an otherwise flat-topped function. The solid black line shows the amplitude envelope formed by summing the Gaussians. (B) The Gaussians used to form the leading and lagging marker envelopes of the no-gap stimulus used as comparison stimulus for the gap stimulus in (A) are shown by the fine and dashed lines, respectively. There are 23 Gaussians in the leading and 23 Gaussians in the lagging marker with 1 ms separating the last Gaussian in the leading marker and the first Gaussian in the lagging marker. Note that an even number of Gaussians is added to the no-gap stimulus when the number of missing Gaussians is an odd number. As a result, the total duration of the no-gap stimulus exceeds the total duration of the gap stimulus by 1 ms. When the number of missing Gaussians was even, the total duration of the gap and no-gap stimuli were identical. (C) Two 20-ms markers formed by six Gaussian envelopes with a standard deviation of $4 \mathrm{~ms}$. Between them is a 16-ms gap, measured between the peak of the last Gaussian of the leading marker and the peak of the first Gaussian of the lagging marker. This gap is produced by the absence of three Gaussians in the middle of the stimulus envelope. The solid line shows the amplitude envelope formed by summing the Gaussians used to define the leading and lagging markers. The blackened portion indicates the difference in area between the central dip in the envelope and an otherwise flat-topped function. (D) The no-gap stimulus corresponding to (C) has eight Gaussians each in the leading and lagging markers. Hence its overall duration is 4 ms longer than the overall duration of the corresponding gap stimulus shown in (C). The solid line shows the amplitude profile for the sum of these 16 Gaussians. (E) In some conditions (primarily those within-channel), some participants were able to detect a gap corresponding to the absence of a single Gaussian between the two markers defining the gap. In these cases, the missing Gaussian was replaced by a Gaussian of reduced amplitude as indicated by the solid line at the center of the stimulus. This produced a dip in the amplitude envelope corresponding to the sum of these Gaussians as indicated in this figure. The blackened portion indicates the difference in area between the central dip in the envelope, and an otherwise flat-topped function. All stimuli were equated with respect to total energy before being presented to the participant. In all analyses, the extent of the gap was measured by the area between the amplitude envelop of the gapped stimulus, and the corresponding flat-topped envelope (shown in black in this figure). Areas were then transformed back into the corresponding gaps using the procedure described in Heinrich and Schneider (2006). ${ }^{1}$ 
(A)

LISTENING CONDITIONS

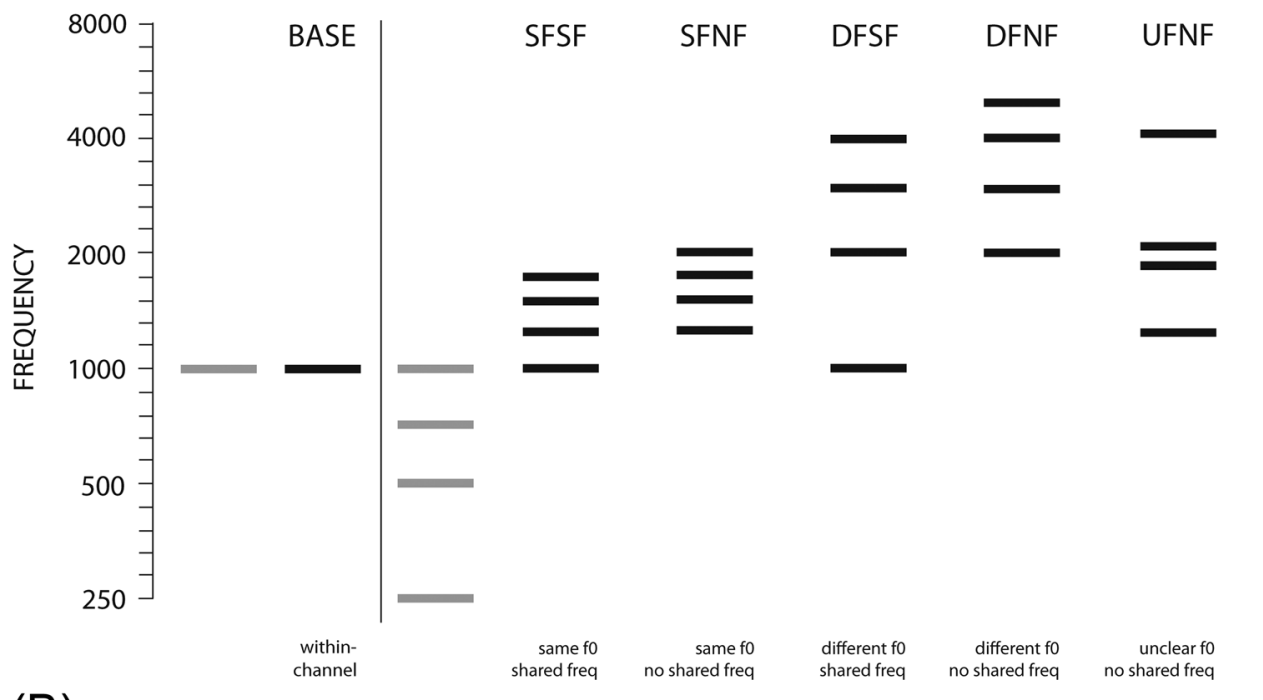

(B)

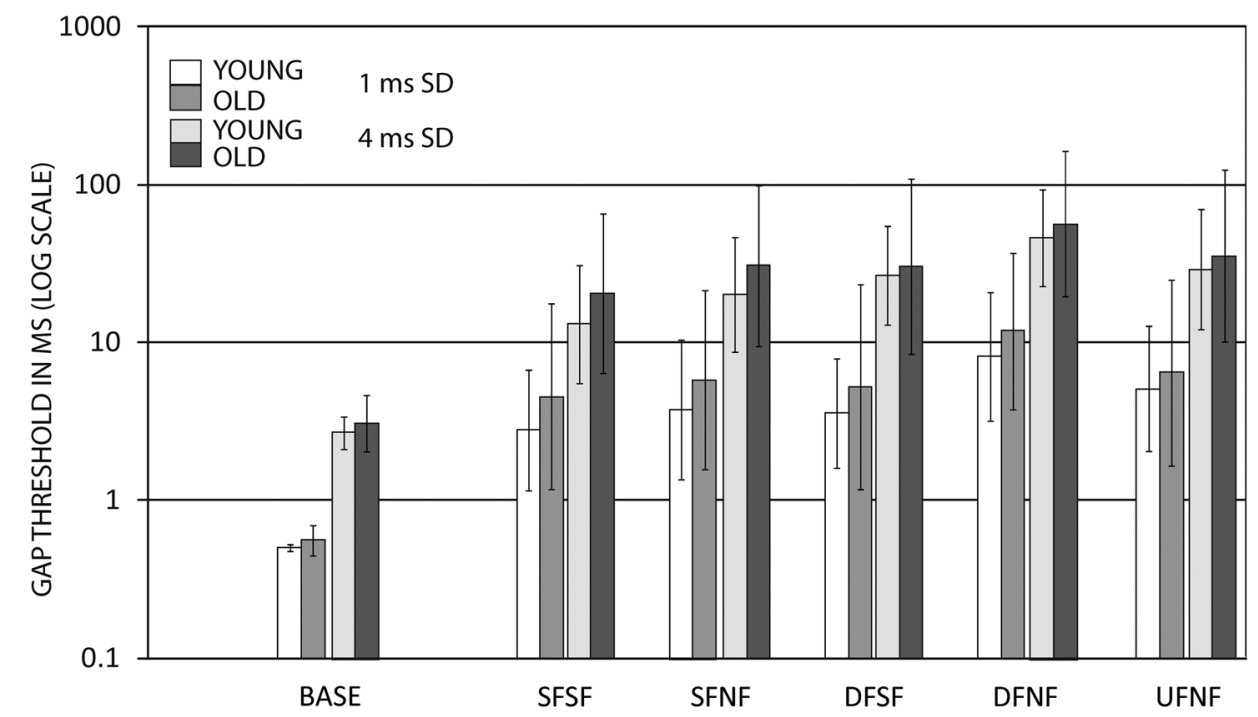

FIG. 2. (A) Spectral content of markers used in all stimulus conditions. In the BASE condition, both leading (gray) and lagging (black) marker consisted of 1-kHz pure tones. In all other conditions, the leading marker consisted of a four-component sound complex with energy at 0.25 , $0.5,0.75$, and $1 \mathrm{kHz}$. The spectral composition of the lagging marker changed between conditions. All conditions were tested with 1- and 4-ms SD Gaussian envelopes. (B) Mean gap detection thresholds for young (white) and older (dark) listeners for 1-ms (lighter shade) and 4-ms (darker shade) SD Gaussian envelope conditions. Error bars depict 1 standard deviation above and below the mean. were re-scaled so that the total energy in a stimulus was equated across all conditions and no energy difference existed between gap and no-gap stimuli. Stimuli were presented to the left ear over TDH-49 headphones in a singlewalled sound-attenuating booth.

\section{Procedure}

A three-down, one-up tracking procedure was used in a 2AFC paradigm to determine the $79.4 \%$ correct point on the psychometric function (Levitt, 1971). At the beginning of each run, the gap size was set to its maximum value of $300 \mathrm{~ms}$. Each trial was initiated by a button press. In each trial, a gap and a no-gap stimulus were presented, randomly assigned to one of two 500-ms long intervals, separated by $100 \mathrm{~ms}$. Each stimulus began $100 \mathrm{~ms}$ into the interval. Listeners were asked to indicate which of the two stimuli contained the gap by pressing one of two buttons. Lights on the button box indicated the beginning and the length of each stimulus interval and provided feedback to the listener about the accuracy of their response. After three consecutive correct responses, the gap size of the stimulus was decreased. After each incorrect response, the gap size was increased. Starting step size was $32 \mathrm{~ms}$. The size of the decrement or increment in gap duration was halved after each reversal until the 1-ms step size in the SD1 condition and the 4-ms step size in the SD4 condition were reached. If listeners perceived gaps constructed with only a single Gaussian missing, gap sizes were decreased further by introducing $25 \%$, $50 \%$, and $75 \%$ reduced amplitude-Gaussians into the gap, which provided additional equivalent gap sizes of $1.5,1$, and $0.5 \mathrm{~ms}$ for SD1 and 6, 4, and $2 \mathrm{~ms}$ for SD4. ${ }^{1}$ There were 12 reversals in total per measurement; the gap-detection threshold for a condition was defined as the mean gap duration at the last eight reversals. The smallest achievable threshold was $0.5 \mathrm{~ms}$ in the SD1 conditions and $2 \mathrm{~ms}$ in the SD4 conditions.

Listeners were not given any practice trials prior to data collection. Testing for each condition was terminated after four thresholds were obtained except for one older and two young listeners, who only provided three thresholds in one condition. In the case of the older listener, this was condition 
TABLE II. (a) Mean ( $\pm 1 \mathrm{SD})$ thresholds in ms for all conditions tested in young and old listeners and displayed in Fig. 2(B).

\begin{tabular}{|c|c|c|c|c|c|c|c|c|c|c|c|c|}
\hline & \multicolumn{6}{|c|}{ SD1 } & \multicolumn{6}{|c|}{ SD4 } \\
\hline & BASE & SFSF & SFNF & DFSF & DFNF & UFNF & BASE & SFSF & SFNF & DFSF & DFNF & UFNF \\
\hline Young mean & 0.51 & 2.86 & 3.93 & 3.67 & 8.44 & 5.24 & 2.81 & 13.57 & 20.75 & 27.70 & 49.45 & 30.30 \\
\hline$-1 \mathrm{SD}$ & 0.49 & 1.18 & 1.41 & 1.64 & 3.29 & 2.10 & 2.22 & 5.70 & 8.93 & 13.39 & 24.26 & 12.61 \\
\hline$+1 \mathrm{SD}$ & 0.53 & 6.93 & 10.97 & 8.21 & 21.67 & 13.07 & 3.55 & 32.31 & 48.22 & 57.31 & 100.80 & 72.84 \\
\hline Old mean & 0.57 & 4.65 & 6.10 & 5.41 & 12.22 & 6.65 & 3.21 & 21.27 & 31.95 & 31.39 & 60.65 & 36.71 \\
\hline$-1 \mathrm{SD}$ & 0.45 & 1.19 & 1.65 & 1.20 & 3.90 & 1.71 & 2.12 & 6.62 & 9.79 & 8.64 & 20.97 & 10.36 \\
\hline$+1 \mathrm{SD}$ & 0.70 & 18.19 & 22.55 & 24.42 & 38.31 & 25.88 & 4.85 & 68.33 & 104.25 & 114.06 & 175.42 & 130.10 \\
\hline
\end{tabular}

(b) Mean (SD) thresholds for all conditions displayed in Fig. 3.

\begin{tabular}{|c|c|c|c|c|}
\hline & Within-channel (BASE1) & Between-channel (1-2/2-1 kHz) (Heinrich and Schneider, 2006) & SFSF(SD1) & $\mathrm{DFNF}(\mathrm{SD} 1)$ \\
\hline \multirow[t]{3}{*}{ Young } & 0.51 & 2.88 & 2.86 & 8.44 \\
\hline & 0.49 & 1.82 & 1.18 & 3.29 \\
\hline & 0.53 & 4.55 & 6.93 & 21.67 \\
\hline \multirow[t]{3}{*}{ Old } & 0.57 & 4.01 & 4.65 & 12.22 \\
\hline & 0.45 & 2.08 & 1.19 & 3.90 \\
\hline & 0.70 & 7.76 & 18.19 & 38.31 \\
\hline
\end{tabular}

DFSF (SD1), for one young listener, it was condition DFSF (SD4), and for another one, it was SFSF (SD1). The threshold for a condition was calculated as the geometric mean of the three lowest estimates. Listeners usually came in for four testing sessions of $1.5 \mathrm{~h}$ each. Conditions were blocked by envelope shape with one half of participants listening to SD1 first. Within the SD block, the baseline condition was always tested first. The order of the remaining conditions was randomized with the provision that all four runs of one condition were tested within the same session. The only exception was one older adult whose BASE (SD1) test occurred before all the SD4 conditions were completed.

\section{RESULTS AND DISCUSSION}

Figure 2(B) and Table II(a) present the mean gapdetection thresholds in milliseconds for young and old listeners for all 12 stimulus conditions. Gap thresholds were substantially higher for SD4 than for SD1 conditions. There also was an effect of f0. Gap detection thresholds were smaller when the leading and lagging markers shared the same fo than when the f0 changed from the first to second marker or when the $\mathrm{fO}$ in the second marker resulted in an unclear pitch. In addition, gap detection thresholds were smaller when harmonic overlap (a shared frequency) existed between the leading and lagging marker. Last, while thresholds were similar for young and old listeners, the results showed a small but consistent advantage for young listeners.

All statistical analyses were performed on the area difference between gap and no-gap envelopes, but the area differences were converted back into equivalent gap durations for the graphical display. ${ }^{1}$ Due to significant heterogeneity between groups, all thresholds were log-transformed $\left(\log _{10}\right)$ before being submitted to statistical analyses to fulfill normality and homoscedasticity assumptions of the analysis of variance (ANOVA) model. Post hoc tests were corrected for family-wise Type-I error rate using the Sidak equation (Sidak, 1967), which provides the same type I error protection as Bonferroni with a higher statistical power (Abdi, 2007).

\section{A. The contribution of pitch, harmonic overlap, envelope, and age to gap detection}

The four clear-pitch lagging-marker conditions, which either kept f0 constant between leading or lagging marker (SFSF, SFNF) or changed it between markers (DFSF, DFNF), were subjected to a f0 change (yes, no) by age (young, old), by spectral overlap (yes, no), by envelope shape (SD1, SD4) mixed-measures ANOVA and showed main effects for f0 change (no change $<$ change), overlap (overlap $<$ no overlap), and envelope $(\mathrm{SD} 1<\mathrm{SD} 4)$ as well as interactions between $\mathrm{f0}$ change and overlap and between f0 change, overlap and envelope. Neither the main effect of age $\left[F(1,38)=2.07, \mathrm{MSE}=1.33, p=0.16, \eta^{2}=0.05\right]$, nor any interaction involving age reached significance. All but two interactions with age (age $\times$ envelope; age $\times$ f0 change) had $F$ values $<1$. Statistical details for this and the following analyses in this section are detailed in Table III.

Note that although the ANOVA provided no statistical evidence for an age difference, Fig. 2(B) shows higher gap-detection thresholds for older than younger listeners in all conditions. In a binomial distribution of outcomes (positive versus negative age differences), this pattern is associated with a probability of chance occurrence of $p(12)_{N=12}<0.001$. Therefore we argue that the data show a consistent, if small, age difference. The interaction between f0 change and overlap occurred because the removal of spectral overlap was particularly detrimental to gap detection when it co-occurred with a change of f0 between markers.

To investigate the three-way interaction among fo change, spectral overlap, and envelope shape, we analyzed the data separately for all conditions in which f0 remained unchanged (SFSF, SFNF) and in which f0 changed between markers (DFSF, DFNF). We also collapsed over the two age groups as no interaction between age and any experimental 
TABLE III. (a) Significant effects for the ANOVAs conducted in Sec. III A. Significant effects for an f0 change (change, no change) $\times$ age (young, old)$\times$ spectral overlap $($ yes, no $) \times$ envelope shape $(1 \mathrm{SD}, 4 \mathrm{SD}) \times$ ANOVA.

\begin{tabular}{lrrrrr}
\hline \hline Effect & $(\mathrm{df1}, \mathrm{df2})$ & $\mathrm{F}$ & $\mathrm{MSE}$ & $p$ & $\eta^{2}$ \\
\hline f0 change & 1,38 & 60.06 & 0.10 & $<0.001$ & 0.61 \\
Overlap & 1,38 & 145.98 & 0.04 & $<0.001$ & 0.79 \\
Envelope & 1,38 & 363.11 & 0.15 & $<0.001$ & 0.91 \\
f0 change $\times$ overlap & 1,38 & 14.85 & 0.04 & $<0.001$ & 0.28 \\
f0 change $\times$ overlap $\times$ envelope & 1,38 & 5.99 & 0.02 & 0.02 & 0.14
\end{tabular}

(b) Significant effects for a spectral overlap (yes, no) $\times$ envelope shape (SD1, SD4) ANOVA restricted to the same f0 conditions (SFSF, SFNF).

\begin{tabular}{lrrrrr}
\hline Overlap & 1,39 & 44.87 & 0.03 & $<0.001$ & 0.54 \\
Envelope & 1,39 & 314.89 & 0.08 & $<0.001$ & 0.89
\end{tabular}

(c) Significant effects for a spectral overlap (yes, no) $\times$ envelope shape (SD1, SD4) ANOVA restricted to the different f0 conditions (DFSF, DFNF).

\begin{tabular}{lrrrrr}
\hline Overlap & 1,39 & 105.29 & 0.05 & $<0.001$ & 0.73 \\
Envelope & 1,39 & 262.54 & 0.11 & $<0.001$ & 0.87 \\
Overlap $\times$ envelope & 1,39 & 6.80 & 0.03 & 0.013 & 0.15
\end{tabular}

(d) Significant effects for an ANOVA where the factors are the different f0 conditions (SFNF, DFNF, UFNF) and envelope shape (SD1, SD4).

\begin{tabular}{lrrrrr}
\hline Envelope & 1,39 & 313.56 & 0.12 & $<0.001$ & 0.89 \\
F0 condition (SFNF, DFNF, UFNF) & 2,78 & 46.65 & 0.06 & $<0.001$ & 0.55 \\
\hline
\end{tabular}

factor had been found. For the two conditions for which f0 remained unchanged (SFSF/SFNF), a spectral overlap (yes, no) by envelope shape (SD1, SD4) repeated-measures ANOVA showed main effects of overlap (overlap $<$ no overlap) and envelope $(\mathrm{SD} 1<\mathrm{SD} 4)$, but no interaction between the two factors $[F(1,39)<1]$. When f0 changed (DFSF/ DFNF), the same ANOVA showed main effects of overlap (overlap $<$ no overlap), and envelope $(\mathrm{SD} 1<\mathrm{SD} 4)$, and a significant interaction. The interaction occurred because the difference in gap thresholds between conditions with and without frequency overlap was bigger for SD1 compared with SD4 conditions.

In addition to the conditions described in the preceding text in which the f0 and overtone structure of the markers were those which have been shown to give rise to clear pitch (Plomp, 1967; Terhardt, 1974), we also investigated another condition in which the second marker was one in which no clear pitch was present (UFNF). To investigate the role of pitch clarity, we compared the condition in which marker pitch changed from a clear pitch in the leading marker to an unclear pitch in the lagging marker (UFNF) to those conditions with clear lagging marker pitches of either the same (SFNF) or a different f0 (DFNF). An f0 condition (SFNF, DFNF, UFNF) $\times$ envelope (SD1, SD4) repeated-measures ANOVA showed main effects of fo condition $(\mathrm{DFNF}>\mathrm{UFNF}>\mathrm{SFNF})$ and envelope $(\mathrm{SD} 1<\mathrm{SD} 4)$, but no interaction $[(F(2,78)<1]$. It was easiest to discriminate gaps when both markers had the same pitch. However, following this, it was easier to discriminate gaps when the lagging marker had no clear pitch than when the lagging marker had a clearly different pitch from the leading marker. Although there was an effect of envelope, it was the same across all three $\mathrm{f} 0$ conditions (no interaction).

\section{B. Age effects and marker complexity}

The leading and lagging markers in a gap detection task can differ with respect to their spectral composition, periodicity, and the degree to which the discrimination can be based on within- as opposed to between-channel comparisons. To evaluate how age affects within- versus betweenchannel gap detection using spectrally simple periodic stimuli (pure tones), we compared SD1 envelope results from the present study with the 20-ms marker duration results from Heinrich and Schneider (2006), which used the same test population, data collection methods, and technique of marker envelope construction for gaps defined between pure tones. We were particularly interested in their betweenchannel conditions in which pure tones either increased from 1 to $2 \mathrm{kHz}$ or decreased from 2 to $1 \mathrm{kHz}$ between markers. Thresholds of these between-channel conditions at 20-ms marker duration were averaged and displayed together with other relevant conditions in Table II(b) and Fig. 3. Pure-tone between-channel thresholds were compared to the $1-\mathrm{kHz}$ pure tone, within-channel condition [BASE(SD1)] of the current study. In this comparison, marker complexity refers to the availability of a within-channel comparison for spectrally simple stimuli. A marker complexity (between- versus within-channel) by age (young, old) ANOVA with both factors between-subjects (as they came from two different studies) showed main effects of marker complexity (withinchannel $<$ between-channel) and age (younger $<$ older), and a significant interaction. The interaction occurred because the effect of age was smaller in the within-channel than in the between-channel condition although age-differences were significant in both [within-channel: $t(38)=2.17$, $p=0.04$; between-channel: $t(46)=2.88, p=0.006]$. Further details of statistical analyses are displayed in Table IV.

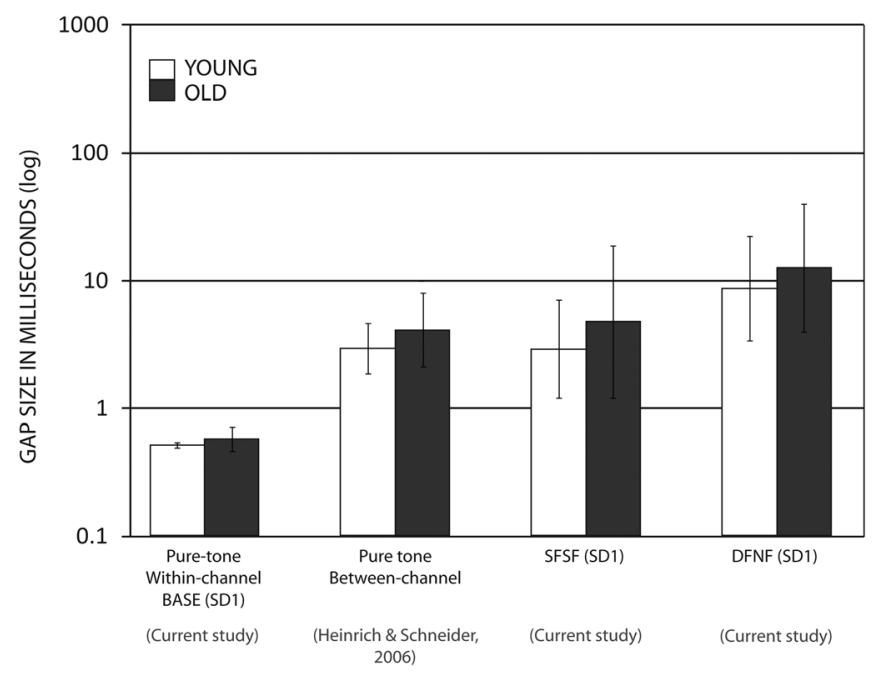

FIG. 3. Mean gap detection thresholds for young (white) and older (dark) listeners for 1-ms SD Gaussian envelope conditions for the current study (within-channel and complex sound) and for Heinrich and Schneider (2006). Error bars depict 1 standard deviation above and below the mean. 
TABLE IV. (a) Significant effects for the ANOVAs conducted in Sec. III B. Significant effects for a comparison of within-channel to between-channel, pure-tone gap detection in a marker complexity (within-channel, betweenchannel) $\times$ age (young, old) ANOVA.

\begin{tabular}{lcrrc}
\hline \hline Effect & $($ df1,df2 $)$ & F & MSE & \multicolumn{1}{c}{$p$} \\
\hline Marker complexity & 1,84 & 554.23 & 0.04 & $<0.001$ \\
Age & 1,84 & 9.79 & 0.04 & 0.002 \\
Marker complexity $\times$ age & 1,84 & 3.84 & 0.04 & 0.05
\end{tabular}

(b) Significant effects for a comparison of within-channel pure tone gap detection to gap detection when the complex tone leading and lagging markers have the same $\mathrm{f0}(250 \mathrm{~Hz})$ and share a common frequency $(1 \mathrm{kHz})$ in a marker complexity [BASE(SD1), $\operatorname{SFSF}(\mathrm{SD} 1)] \times$ age (young, old) ANOVA.

\begin{tabular}{lrrrc}
\hline Marker complexity & 1,38 & 177.65 & 0.104 & $<0.001$ \\
Age & 1,38 & 4.85 & 0.14 & 0.03
\end{tabular}

(c) Significant effects for a comparison of between-channel pure tone gap detection to gap detection when the complex tone leading and lagging markers have no spectral overlap and do not have the same F0 $(250 \mathrm{~Hz})$ in a marker complexity [between-channel pure tone, DFNF $(\mathrm{SD} 1)] \times$ age (young, old) ANOVA.

\begin{tabular}{lrrrc}
\hline Marker complexity & 1,84 & 62.93 & 0.13 & $<0.001$ \\
Age & 1,84 & 6.34 & 0.13 & 0.01
\end{tabular}

(d) Significant effects for an age (young, old) $\times$ change type (periodicity, pitch) $\times$ overlap (yes, no) ANOVA.

\begin{tabular}{lrrrc}
\hline Overlap & 1,68 & 167.03 & 0.05 & $<0.001$ \\
Change type & 1,68 & 47.87 & 0.21 & $<0.001$ \\
Age & 1,68 & 16.03 & 0.21 & $<0.001$ \\
Overlap $\times$ change type & 1,68 & 5.74 & 0.05 & 0.02 \\
\hline \hline
\end{tabular}

We also compared within-channel pure tone gapdetection thresholds in BASE1(SD1) to the complex tone SFSF (SD1) conditions where the leading and lagging markers had the same fo $(250 \mathrm{~Hz})$ and a common frequency component of $1 \mathrm{kHz}$. Note that SFSF was different from a pure within-channel condition because, except for the common frequency component at $1 \mathrm{kHz}$, none of the other frequency components in the two markers overlapped with one another, and the fundamental frequency of the two tonal markers $(250 \mathrm{~Hz})$ was different from the frequency of spectral overlap $(1 \mathrm{kHz})$. Note that both BASE and SFSF permit a within-channel comparison. However, because the markers in BASE are identical pure tones, this within-channel comparison is based on spectrally simple markers, whereas the within-channel comparison in SFSF occurs at the same frequency $(1 \mathrm{kHz})$, the two markers differ in spectral content, with their f0 at $250 \mathrm{~Hz}$ rather than $1 \mathrm{kHz}$. An analysis of these two conditions [BASE(SD1) vs SFSF(SD1)] showed main effects of marker complexity (pure-tone markers $<$ complex-tone markers) and age (young $<$ old), but no significant interaction between the two.

A third analysis compared pure-tone between-channel gap detection thresholds (Heinrich and Schneider, 2006) and DFNF (SD1) thresholds (complex tones with different f0s and no spectral overlap) from the current study in both young and old listeners. In both of these conditions, there was a change in $\mathrm{f} 0$ between the leading and lagging markers ( 1 and $2 \mathrm{kHz}$ in the pure tone between-channel and $250 \mathrm{~Hz}$ to
$1 \mathrm{kHz}$ in DFNF) with no spectral overlap. This analysis found main effects of marker complexity (pure-tone between-channel threshold $<$ complex-tone between-channel) and age (young < old) but no interaction between the two. Older adults had higher gap-detection thresholds than younger adults in these three situations in which marker complexity was varied. However, the only situation in which there was evidence of a differential age effect was that between within- and between-channel pure-tone markers. This suggests that older adults were not particularly disadvantaged relative to younger adults as marker complexity increased.

Last, the current study aimed to shed further light on results of Pichora-Fuller et al. (2006) by comparing, in younger and older adults, the effects of a change in periodicity between the leading and lagging markers to a change in fo between two periodic markers both when there is spectral overlap between the two markers and when the two markers do not overlap spectrally. The comparison to the PichoraFuller et al. study could be made because the stimuli in the Pichora-Fuller et al. study were constructed using the same technique of Gaussian markers envelopes that was also used in the current study. Note, however, that the Gaussians in the Pichora-Fuller et al. study had a standard deviation of $0.5 \mathrm{~ms}$ and that the closest comparable stimulus duration was $40 \mathrm{~ms}$. From the Pichora-Fuller et al. study, two conditions were of particular interest because the markers differed with respect to periodicity. In one of these conditions, the leading marker was the consonant [s], and the lagging marker was the vowel [u]. Here there was a large degree of spectral overlap between the two markers. In the second condition, the leading marker was broadband noise $(1-6 \mathrm{kHz})$ and the lagging marker was a $500 \mathrm{~Hz}$ pure tone (no spectral overlap between the two markers). These two conditions could be compared to the DFSF condition of the current study, which had a change in $\mathrm{f} 0$ from the leading to lagging marker along with some degree of spectral overlap between the two markers and the DFNF condition, which had a change in f0 without any spectral overlap between the two markers. From the current study only SD1 conditions were used for the comparison. The Table $\mathrm{V}$ displays means in $\mathrm{ms}$ and in log-scaled values for these four conditions (The raw data for the periodicity change conditions were supplied by PichoraFuller et al.).

Subjecting the log-transformed data in Table $\mathrm{V}$ to a two age (young, old) by two change type (periodicity, pitch) by two overlap (yes, no) mixed-measures ANOVA with age and change type as between- and overlap as within-subject factors, found main effects of overlap (overlap $<$ no overlap), change type (pitch change $<$ periodicity change), and age (young $<$ old), and an interaction between overlap and change type where a change from overlap to no overlap led to a larger increase in threshold when there was a corresponding change in periodicity than when the change in overlap was coupled with a change in pitch. None of the interactions involving age were significant (details of the statistical analyses are displayed in Table IV). Periodicity changes between markers had a greater adverse effect on gap detection thresholds than pitch changes, particularly 
TABLE V. Gap-detection thresholds for younger and older listeners for marker conditions with a change of periodicity or pitch and with or without partial overlap between leading and lagging markers. Values are given in mean (SD) $\mathrm{ms}$ and log values. The periodicity change values are taken from Pichora-Fuller et al. (2006) while the pitch change values are taken from the current study. In particular, periodicity change with spectral overlap corresponds to the /s-u/40ms condition of Pichora-Fuller et al:; periodicity change without spectral overlap corresponds to their broadband noise 40-ms condition; pitch change with spectral overlap corresponds to the DFSF (SD1) condition in current study; pitch change without overlap corresponds to the current study's DFNF (SD1) condition.

\begin{tabular}{|c|c|c|c|c|c|}
\hline & & \multicolumn{2}{|c|}{ Younger listeners } & \multicolumn{2}{|c|}{ Older listeners } \\
\hline & & With overlap & Without overlap & With overlap & Without overlap \\
\hline \multirow[t]{2}{*}{ Periodicity change } & $\mathrm{ms}$ & $10.70(7.25)$ & 33.88 (19.69) & $29.03(21.71)$ & 109.75 (69.13) \\
\hline & $\log _{10}$ & $0.96(0.24)$ & $1.46(0.24)$ & $1.35(0.32)$ & $1.94(0.34)$ \\
\hline \multirow[t]{2}{*}{ Pitch change } & $\mathrm{ms}$ & $3.67(2.47)$ & $8.44(12.38)$ & $5.41(21.19)$ & $12.22(24.78)$ \\
\hline & $\log _{10}$ & $0.59(0.25)$ & $1.01(0.36)$ & $0.82(0.54)$ & $1.15(0.42)$ \\
\hline
\end{tabular}

when there was no spectral overlap between markers. Remarkably, this pattern of results was the same for young and old listeners when thresholds were measured on a logtransformed scale indicating that older listeners did not find it differentially more difficult to detect gaps when there was change in periodicity between markers than when there was a change in pitch. However, note that there was an overall age effect in that older adults had larger gap-detection thresholds overall than did younger adults.

\section{Correlation with pure-tone thresholds}

Given that older listeners had significantly higher audiometric thresholds than younger listeners on almost all frequencies used for the gap stimuli in this study, it is conceivable that the age differences found in gap detection would covary with hearing loss. We investigated this possibility by computing Pearson product-moment correlations between gapdetection thresholds for a particular stimulus condition and their average hearing level across all frequencies represented in the stimulus (Table VI). In the BASE conditions, the gap thresholds were related to the pure-tone threshold at $1 \mathrm{kHz}$ in the left ear. In the four SF_ conditions (SD1 and SD4), gap thresholds were related to the pure-tone average threshold of $0.25,0.5,1$, and $1.5 \mathrm{kHz}$ (PTA_low). In the four DF_ conditions and the two UF conditions (SD1 and SD4), gap thresholds were related to the pure-tone average threshold of 0.25 , $0.5,1$ and $1.5,2,3,4 \mathrm{kHz}$ (PTA_high). The results showed that hearing thresholds did not reliably correlate with gap detection thresholds. In particular, only 1 of the 24 correlations in Table VI reached an uncorrected $\alpha=0.05$ level of significance (and that was in young rather than older listeners), which falls within the Type-I error margin of $5 \%$ of the 24 correlation coefficients. Hence there is no evidence that hearing level affected the results.

\section{CONCLUSIONS}

The results of this study indicate that detecting a gap between two tonal markers was affected by the following marker characteristics: f0 change, availability of withinchannel comparisons, and envelope shape. There was also a small effect of age but not of hearing status, at least as long as hearing was reasonably good.

Pitch changes from leading to lagging marker resulted in larger gap-detection thresholds than constant marker pitch (SF_ versus DF_ conditions). Moreover, the increase in gap-detection thresholds was larger when the lagging marker had a clear but different pitch from the leading marker than when the lagging marker had no clear pitch (SFNF, UFNF, and DFNF). Finally, the effects of pitch and spectral overlap interacted such that threshold increases for spectrally nonoverlapping markers were larger when the pitch also differed between markers (SFNF versus DFNF). We also replicated previous findings that slow rise and decay times resulted in larger gap-detection thresholds than fast rise and decay times. This effect cannot be attributed to spectral scatter (Heinrich and Schneider, 2006) but rather indicates that sharp onsets and offsets in the temporal domain facilitate the detection of a gap.

TABLE VI. Correlation (Pearson's $r$ ) between audiometric threshold in dB HL in the left ear and gap size in ms. PTA_low represents the average audiometric threshold of testing frequencies $0.25,0.50,1$, and $1.5 \mathrm{kHz}$ in the left ear. PTA_high represents the average audiometric threshold of testing frequencies 0.25 , $0.50,1,1.5,2,3$ and $4 \mathrm{kHz}$ in the left ear. Only one of 24 correlations between hearing sensitivity and gap threshold reached an uncorrected level of significance of $p<0.05$.

\begin{tabular}{|c|c|c|c|c|c|c|c|c|c|c|c|c|c|}
\hline & & \multicolumn{2}{|c|}{ BASE } & \multicolumn{2}{|c|}{ SFSF } & \multicolumn{2}{|c|}{ SFNF } & \multicolumn{2}{|c|}{ DFSF } & \multicolumn{2}{|c|}{ DFNF } & \multicolumn{2}{|c|}{ UFNF } \\
\hline & & 1 & 4 & 1 & 4 & 1 & 4 & 1 & 4 & 1 & 4 & 1 & 4 \\
\hline \multirow[t]{2}{*}{$1 \mathrm{kHz}$} & Young & 0.02 & 0.09 & & & & & & & & & & \\
\hline & Old & 0.21 & 0.09 & & & & & & & & & & \\
\hline \multirow[t]{2}{*}{ PTA_low } & Young & & & $0.46^{*}$ & 0.41 & 0.22 & 0.16 & & & & & & \\
\hline & Old & & & 0.30 & 0.26 & 0.13 & 0.29 & & & & & & \\
\hline \multirow[t]{2}{*}{ PTA_high } & Young & & & & & & & 0.17 & 0.06 & 0.00 & 0.14 & 0.16 & 0.13 \\
\hline & Old & & & & & & & 0.10 & -0.05 & -0.03 & -0.01 & 0.16 & -0.02 \\
\hline
\end{tabular}

${ }^{*} p<0.05$. 
Marker complexity increased gap detection thresholds. Thresholds were substantially lower for within-channel comparisons of identical pure-tone markers (BASE) than for spectrally complex markers with identical pitches that allow for a within-channel comparison at one frequency (SFSF). Similarly, pure-tone markers with f0 change (making these comparisons completely between-channel with no spectral overlap) produced substantially lower gap-detection thresholds than when complex-tone markers changed f0 and had no spectral overlap (DFNF).

Age differences were present but small for gap detection in complex markers conditions. Moreover, with one exception (a comparison between pure-tone within- and betweenchannel gap detection), we failed to find evidence that the size of the age effect increased with stimulus complexity when analyzing the data using log-thresholds. This finding is a departure from the majority of studies in the literature, which evaluated age differences in thresholds on a linear scale and tended to find increasing age differences for more complex stimuli (e.g., Lister et al., 2002; Pichora-Fuller et al., 2006; Harris et al., 2010). A brief example illustrates how a log transform of the gap-detection thresholds could account for the different conclusions concerning the effects of age on gap detection. A common log difference of 0.3 corresponds to a ratio of 2:1. Now suppose that gapdetection thresholds for pure-tone within-channel comparisons are twice as large for older adults (say $4 \mathrm{~ms}$ ) as for younger adults $(2 \mathrm{~ms})$. If the same logarithmic age difference is maintained when the markers are complex tones differing in f0 and having no spectral overlap, and younger adults' thresholds are $80 \mathrm{~ms}$, then the thresholds for older adults would be $160 \mathrm{~ms}$, a value that is consistent with previous studies in the literature (Lister et al., 2002; Pichora-Fuller et al., 2006). Hence we argue that although there is evidence of an age difference in gap detection, there is no evidence that the size of the age difference is modulated by tonal complexity when thresholds are evaluated on a $\log$ scale. Given that threshold variability tends to increase with stimulus complexity, one could argue that a log-transformation of data in these conditions would be appropriate if data were subsequently treated with parametric analysis tools.

\section{ACKNOWLEDGMENTS}

This research was supported by the Natural Sciences and Engineering Research Council of Canada (Grant No. 9172). We would like to thank Jane Carey and Alham Chelehmalzadeh for assistence with data collection.

\footnotetext{
${ }^{1}$ Consider a sum of Gaussians. We can show that when $m$ Gaussians are removed from the middle of the envelope, the time difference between when the envelope decays to 0.7 of its peak value and when it returns to 0.7 of its peak amplitude is approximately equal to $(m+1) \sigma$. Because the 0.7 position on the amplitude envelope is approximately the 0.5 position on the squared envelope, the time difference between the half-power points on the envelope is approximately equal to $(m+1) \sigma$ for $m$ greater than or equal to 1 . The difference in area, $A$, between the full envelope and the envelope missing $m$ Gaussians is equal to $m \sqrt{2 \pi} \sigma$. Note that the time gap, $t_{g}$ (measured in seconds), created by the $m$ missing Gaussians is $t_{g}=(m+1) \sigma$, for $m$ greater than or equal to 1 . Therefore area is linearly related to time gap by $A=\left(t_{g}-\sigma\right) \sqrt{2 \pi}$ for $t_{g} \geq 2 \sigma, t_{g}$ measured in seconds. When there are no missing Gaussians, the gap duration is defined as
}

0 , and the area is 0 . When there is one missing Gaussian, the gap duration is $2 \sigma$ and the area is $\sqrt{2 \pi} \sigma$. If we now assume that the area is linearly related to gap duration over this range, then area is equal to $(\sqrt{2 \pi} / 2) t_{g}$ measured in seconds, and $0<\mathrm{t}_{\mathrm{g}}<2 \sigma$. All analyses were performed on areas, and means were then converted back to equivalent gap durations using these linear relationships.

Abdi, H. (2007). "The Bonferonni and îSidák corrections for multiple comparisons," in Encyclopedia of Measurement and Statistics, edited by N. J. Salkind (Sage, Thousand Oaks, CA), pp. 103-107.

Alain, C., McDonald, K. L., Ostroff, J. M., and Schneider, B. (2001). "Agerelated changes in detecting a mistuned harmonic," J. Acoust. Soc. Am. 109, 2211-2216.

Bernstein, J. G. W., and Oxenham, A. J. (2006). "The relationship between frequency selectivity and pitch discrimination: Sensorineural hearing loss," J. Acoust. Soc. Am. 120, 3929-3945.

Cacciatore, F., Napoli, C., Abete, P., Marciano, E., Triassi, M., and Rengo, F. (1999). "Quality of life determinants and hearing function in an elderly population: Osservatorio Geriatrico Campano Study Group," Gerontology $\mathbf{4 5}, 323-328$.

Clark, J. G. (1981). "Uses and abuses of hearing loss classification," ASHA 23, 493-500.

Committee on Hearing Bioacoustics and Biomechanics (CHABA) (1988). "Speech understanding and aging," J. Acoust. Soc. Am. 83, 859-895.

Divenyi, P. L., and Danner, W. F. (1977). "Discrimination of time intervals marked by brief acoustic pulses of various intensities and spectra," Percept. Psychophys. 21, 125-142.

Formby, C., and Forrest, T. G. (1991). "Detection of silent temporal gaps in sinusoidal markers," J. Acoust. Soc. Am. 89, 830-837.

Frisina, D. R., and Frisina, R. D. (1997). "Speech recognition in noise and presbycusis: Relations to possible neural mechanisms," Hear. Res. 106, 95-104.

Grose, J. H., Hall, J. W., Buss, E., and Hatch, D. (2001). "Gap detection for similar and dissimilar gap markers," J. Acoust. Soc. Am. 109, 1587-1595. Grube, M., von Cramon, D. Y., and Rubsamen, R. (2003). "Inharmonicity detection: Effects of age and contralateral distractor sounds," Exp. Brain Res. 153, 637-642.

Harris, K. C., Eckert, M. A., Ahlstrom, J. B., and Dubno, J. R. (2010). "Age-related differences in gap detection: Effects of task difficulty and cognitive ability,” Hear. Res. 264, 21-29.

Heinrich, A., and Schneider, B. (2006). "Age-related changes in within- and between-channel gap detection using sinusoidal stimuli," J. Acoust. Soc. Am. 119, 2316-2326.

Leung, A. W. S., Jolicoeur, P., Vachon, F., and Alain, C. (2011). "The perception of concurrent sound objects in harmonic complexes impairs gap detection," J. Exp. Psychol. Hum. Percept. Perform. 37, 727-736.

Levitt, H. (1971). "Transformed up-down methods in psychoacoustics," J. Acoust. Soc. Am. 49, 467-477.

Lister, J., Besing, J., and Koehnke, J. (2002). "Effects of age and frequency disparity on gap discrimination," J. Acoust. Soc. Am. 111, 2793-2800.

Lister, J., and Tarver, K. (2004). "Effect of age on silent gap discrimination in synthetic speech stimuli," J. Speech Lang. Hear. Res. 47, 257-268.

Lister, J. J., Koehnke, J. D., and Besing, J. M. (2000). "Binaural gap duration discrimination in listeners with impaired hearing and normal hearing," Ear Hear. 21, 41-50.

Mohlin, P. (2011). "The just audible tonality of short exponential and Gaussian pure tone bursts," J. Acoust. Soc. Am. 129, 3827-3836.

Moore, B. C. J., and Moore, G. A. (2003). "Discrimination of the fundamental frequency of complex tones with fixed and shifting spectral envelopes by normally hearing and hearing-impaired subjects," Hear. Res. 182, 153-163.

Moore, B. C. J., Vickers, D. A., Plack, C. J., and Oxenham, A. J. (1999). "Inter-relationship between different psychoacoustic measures assumed to be related to the cochlear active mechanism," J. Acoust. Soc. Am. 106, 2761-2778.

Oxenham, A. J. (2000). "Influence of spatial and temporal coding on auditory gap detection," J. Acoust. Soc. Am. 107, 2215-2223.

Phillips, D. P., Taylor, T. L., Hall, S. E., Carr, M. M., and Mossop, J. E. (1997). "Detection of silent intervals between noises activating different perceptual channels: Some properties of 'central' auditory gap detection," J. Acoust. Soc. Am. 101, 3694-3705.

Pichora-Fuller, M. K., Schneider, B. A., Benson, N. J., Hamstra, S. J., and Storzer, E. (2006). "Effect of age on detection of gaps in speech and nonspeech markers varying in duration and spectral symmetry," J. Acoust. Soc. Am. 119, 1143-1155. 
Plomp, R. (1967). "Pitch of complex tones," J. Acoust. Soc. Am. 41 $1526-1533$.

Schneider, B. A., and Hamstra, S. J. (1999). "Gap detection thresholds as a function of tonal duration for younger and older listeners," J. Acoust. Soc. Am. 106, 371-380.

Schneider, B. A., and Pichora-Fuller, M. K. (2001). "Age-related changes in temporal processing: Implications for speech perception," Semin. Hear. 22, 227-240.

Schneider, B. A., Pichora-Fuller, M. K., and Daneman, M. (2010). "Effects of senescent changes in audition and cognition on spoken language comprehension," in The Aging Auditory System, edited by S. GordonSalant, R. D. Frisina, A. N. Popper, and R. R. Fay (Springer Verlag, New York), pp. 167-210.

Schneider, B. A., Pichora-Fuller, M. K., Kowalchuk, D., and Lamb, M. (1994). "Gap detection and the precedence effect in young and old adults," J. Acoust. Soc. Am. 95, 980-991.
Schneider, B. A., Speranza, F., and Pichora-Fuller, M. K. (1998). "Agerelated changes in temporal resolution: Envelope and intensity effects," Can. J. Exp. Psychol. 52, 184-191.

Shailer, M. J., and Moore, B. C. J. (1987). "Gap detection and the auditory filter: Phase effects using sinusoidal stimuli," J. Acoust. Soc. Am. 81, 1110-1117.

Sidak, Z. (1967). "Rectangular confidence regions for the means of multivariate normal distributions," J. Am. Statist. Assoc. 62, 626-633.

Strouse, A., Ashmead, D. H., Ohde, R. N., and Grantham, D. W. (1998). "Temporal processing in the aging auditory system," J. Acoust. Soc. Am. 104, 2385-2399.

Terhardt, E. (1974). "Pitch, consonance, and harmony," J. Acoust. Soc. Am. 55, 1061-1069.

Tyler, R. S., Wood, E. J., and Fernandes, M. (1983). "Frequency resolution and discrimination of constant and dynamic tones in normal and hearingimpaired listeners," J. Acoust. Soc. Am. 74, 1190-1199. 